\title{
Parallel Multistage Decision Feedback Equalizer for Single-Carrier Layered Space-Time Systems in Frequency-Selective Channels
}

\author{
Jing Xu \\ National Mobile Communications Research Lab, Southeast University, Nanjing 210096, China \\ Email:xujing@seu.edu.cn \\ Haifeng Wang \\ Nokia Mobile Phones, Elektroniikkatie 3, 90571 Oulu, Finland \\ Email: haifeng.wang@nokia.com

\section{Shixin Cheng} \\ National Mobile Communications Research Lab, Southeast University, Nanjing 210096, China \\ Email: sxcheng@seu.edu.cn \\ Ming Chen \\ National Mobile Communications Research Lab, Southeast University, Nanjing 210096, China \\ Email: chenming@seu.edu.cn
}

Received 24 February 2003; Revised 8 January 2004

\begin{abstract}
Space-time transmission techniques can greatly increase the spectral efficiency. In this paper, a parallel multistage decision feedback equalizer (PMDFE) is proposed for single-carrier layered space-time systems with a fixed cyclic prefix over frequency-selective channels. It is composed of a parallel interference canceller, a multiple-input single-output decision feedback equalizer (MISODFE), and a linear combiner. The soft output of the MISO-DFE is linearly combined with the previous tentative soft decision. In addition, an algorithm is proposed to obtain tentative soft and hard decisions for initializing the equalizer. The initializing complexity of the PMDFE is lower than that of MIMO-OFDM. Simulation results show that the PMDFE outperforms MIMO-OFDM and previously existing equalizers for single-carrier layered space-time systems.
\end{abstract}

Keywords and phrases: MIMO, OFDM, PIC, DFE, single carrier.

\section{INTRODUCTION}

Conventional technique to mitigate intersymbol interference (ISI) is equalization in time domain (TD). For broadband transmission, the complexity of equalization in TD will be very high. Orthogonal frequency division multiplexing (OFDM) transmission [1] that can be considered as a onetap linear equalizer is a promising technique to eliminate ISI. However, the high peak-to-average power ratio will degrade its performance. Another technique to eliminate ISI is singlecarrier modulation with a cyclic prefix $(\mathrm{CP})$ and frequency domain (FD) equalization. Frequency domain linear equalizers (FDLE) proposed in $[2,3,4]$ make this technique a candidate for broadband transmission. Recently, a new decision feedback equalizer (DFE) is proposed in $[5,6]$ for single- carrier system with a fixed CP in single-input single-output (SISO) frequency-selective channel. Its forward filter is performed in FD and the feedback filter is in TD. A fixed CP is utilized to initialize the feedback filter. This scheme can achieve better performance than the FDLE. Moreover, the complexity of this scheme does not increase with RMS delay spread. Results in [7] show that the fixed CP can be used for channel estimation and synchronization.

For multimedia services, more bandwidth is demanded. Space-time transmission techniques $[8,9]$ can greatly increase the spectral efficiency. Layered space-time receivers with interference cancellation are proposed [10] to mitigate ISI and cochannel interference (CCI) for frequency-selective channels. However, the complexity of space-time processing in TD increases with RMS delay spread. Multiple-input 
multiple-output OFDM (MIMO-OFDM) scheme [11] is a satisfactory solution not only to mitigate ISI but also to obtain high spectral efficiency. The MIMO decision feedback equalizer (MIMO-DFE) [12] with its forward filter performed in FD and feedback filter implemented in TD is proposed for single-carrier layered space-time systems. However, the complexity of the MIMO-DFE is too high to be implemented. Meanwhile, a linear equalizer along with one stage parallel interference cancellation (PIC) in FD (FDLEPIC) is also proposed in [12]. Since PIC in [12] is equivalent to a DFE in space domain (SD), the FDLE-PIC can be considered as a DFE in SD combined with a linear equalizer in FD.

In this paper, a multiple-input single-output decision feedback equalizer (MISO-DFE) with its forward and feedback filter performed in FD and TD, respectively, is proposed for equivalent single-input multiple-output channels. Then a parallel multistage decision feedback equalizer (PMDFE) is proposed for single-carrier layered space-time systems with a fixed CP. It is composed of a parallel interference canceller, a MISO-DFE, and a linear combiner. The soft output of MISODFE is linearly combined with the previous tentative soft decision. The proposed PMDFE is equivalent to the DFE in SD combined with a MISO-DFE in FD and TD. Moreover, partial parallel interference cancellation (PPIC) idea stemming from multiuser detector [13] is introduced to reduce the effect of error propagation. The complexity of the PMDFE does not increase with RMS delay spread and the performance of the PMDFE is better than the MIMO-OFDM and the FDLE-PIC. In addition, an algorithm is proposed to obtain tentative soft and hard decisions for the initialization of the PMDFE.

The paper is organized as follows. In Section 2, channel models and single-carrier layered space-time systems are introduced. Then the PMDFE is derived in Section 3. In Section 4, we formulate an algorithm to obtain tentative soft and hard decisions for the initialization of the PMDFE. In Section 5, the complexity of existing schemes is compared in terms of complex multiplication. Finally, in Section 6, the performance of the proposed equalizer is compared with existing schemes through computer simulations.

\section{CHANNEL MODEL AND SINGLE-CARRIER SYSTEMS}

The layered space-time architecture is given in Figure 1. As shown in Figure 2, the $k$ th transmission block from the $i$ th $\mathrm{Tx}$ is

$$
\begin{aligned}
Q_{i}[k]= & {\left[s_{i}[k(T+L)], s_{i}[k(T+L)+1], \ldots,\right.} \\
& \left.s_{i}[(k+1)(T+L)-1]\right] \\
= & {\left[d_{i}[k T], d_{i}[k T+1], \ldots, d_{i}[k T+T-1],\right.} \\
& \left.\quad \mathrm{PN}_{i, 0}, \mathrm{PN}_{i, 1}, \ldots, \mathrm{PN}_{i, L-1}\right], \quad \text { for } i=1,2, \ldots, M,
\end{aligned}
$$

where $L$ is the length of $\mathrm{CP}$, the last $L$ symbols are pseudonoise $(\mathrm{PN})$ sequence, and $T$ denotes the number of transmitted symbols in one data block. These data blocks are

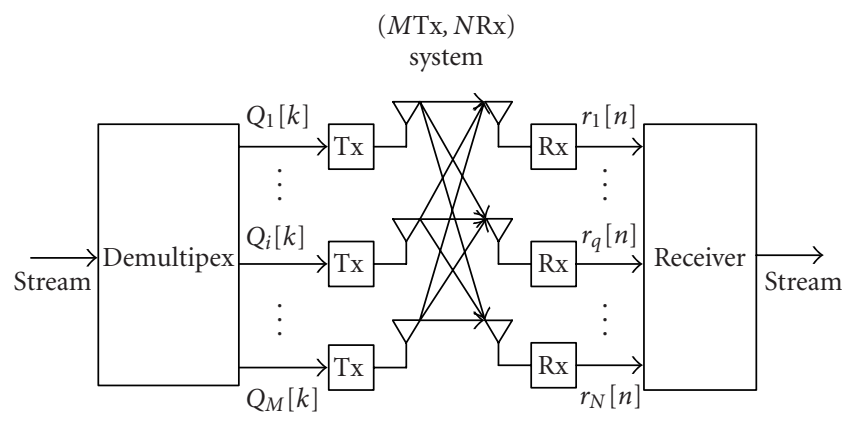

FIGURE 1: Layered space-time architecture.

simultaneously transmitted by $M$ transmitters. In addition, the same PN sequence is transmitted before the first data block. This technique that forces linear convolution into circular convolution was first proposed in [14]. PN sequences are fixed, known at the receiver, and suitable for use with a DFE. The conventional technique [1] is to add a CP in front of a data block. However, the CP of the conventional technique changes with data block. The received signal from the $q$ th receive antenna at time instant $n$ is

$$
\begin{aligned}
& r_{q}[k(T+L)+n] \\
& =\sum_{i=1}^{M} \sum_{l=0}^{L_{i, q}-1} s_{i}[k(T+L)+n-l] h_{i, q}[l]+v_{q}[k(T+L)+n], \\
& \quad n=-L,-(L-1), \ldots, T+L-1, q=1, \ldots, N,
\end{aligned}
$$

where $L_{i, q}$ is the memory length of the channel from the $i$ th transmit antenna to the $q$ th receive antenna, $h_{i, q}[l]$ denotes the $l$ th tap of the channel corresponding to the $i$ th transmit antenna and the $q$ th receive antenna, and $v_{q}[n]$ denotes the complex additive white Gaussian noise with variance $\sigma_{v}^{2}$ at the $q$ th receive antenna. For convenience, we will drop the index $k$ in the following discussion. Discarding the CP, the $(T+L)$-sized DFT of the received signal at the $q$ th receive antenna is

$$
R_{q}[f]=\sum_{i=1}^{M} S_{i}[f] H_{i, q}[f]+V_{q}[f], \quad f=0,1, \ldots, T+L-1,
$$

where

$$
\begin{aligned}
& S_{i}[f]=\sum_{n=0}^{T+L-1} s_{i}[n] e^{-j 2 \pi n f /(T+L)}, \\
& H_{i, q}[f]=\sum_{l=0}^{L_{i, q}} h_{i, q}[l] e^{-j 2 \pi l f /(T+L)} .
\end{aligned}
$$

In $\mathrm{FD}$, the input-output relation can be expressed in vector form as follows:

$$
\mathbf{R}[f]=\mathbf{H}[f] \mathbf{S}[f]+\mathbf{V}[f],
$$




\begin{tabular}{l|l|l|l|l}
\hline$d_{i}[(k-1) T+T-1]$ & $\mathrm{PN}_{i, 0}, \ldots, \mathrm{PN}_{i, L-1}$ & $d_{i}[k T], d_{i}[k T+1], \ldots, d_{i}[k T+T-1]$ & $\mathrm{PN}_{i, 0}, \ldots, \mathrm{PN}_{i, L-1}$ & $d_{i}[(k+1) T]$ \\
\hline
\end{tabular}

FIgURE 2: The transmission format of the $k$ th data block.

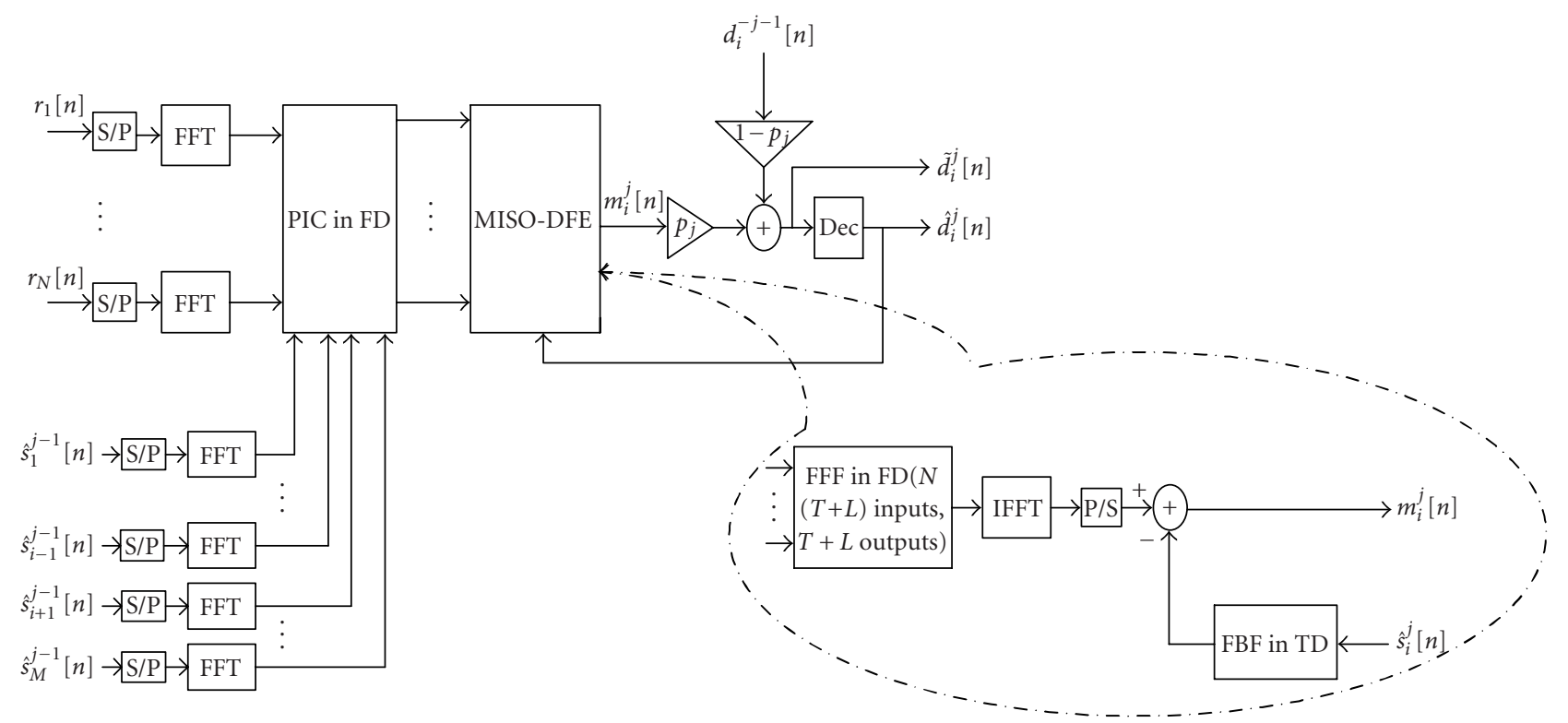

FIgURE 3: The $j$ th detection stage of the $i$ th substream.

where

$$
\begin{gathered}
\mathbf{R}[f]=\left[\begin{array}{c}
R_{1}[f] \\
\vdots \\
R_{N}[f]
\end{array}\right], \\
\mathbf{H}[f]=\left[\begin{array}{ccc}
H_{1,1}[f] & \cdots & H_{1, M}[f] \\
\vdots & \ddots & \vdots \\
H_{N, 1}[f] & \cdots & H_{N, M}[f]
\end{array}\right], \\
\mathbf{S}[f]=\left[\begin{array}{c}
S_{1}[f] \\
\vdots \\
S_{M}[f]
\end{array}\right], \\
\mathbf{V}[f]=\left[\begin{array}{c}
V_{1}[f] \\
\vdots \\
V_{N}[f]
\end{array}\right] .
\end{gathered}
$$

\section{RECEIVER FOR SINGLE-CARRIER LAYERED SPACE-TIME SYSTEMS IN FREQUENCY-SELECTIVE CHANNELS}

In this section, we assume that initial tentative soft and hard decisions for the transmitted symbols have been obtained. In the following section, an algorithm will be introduced to implement this initialization. As shown in Figure 3, with the tentative hard decision at the $(j-1)$ th stage and parallel canceling the interference to the $i$ th substream in FD, the mod- ified received signal for the $i$ th substream in FD at the $f$ th subchannel can be expressed as follows:

$$
\mathbf{R}^{i}[f]=\mathbf{R}[f]-\sum_{t=1, t \neq i}^{M} \hat{S}_{t}^{j-1}[f](\mathbf{H}[f])_{t},
$$

where $(\mathbf{H}[f])_{t}$ denotes the $t$ th column of channel matrix and $\hat{S}_{t}^{j-1}[f]$ is the DFT of the tentative hard decision $\hat{s}_{t}^{j-1}[n]$ for the $t$ th substream at the $(j-1)$ th stage. As shown in Figure 3, applying the weight vector of the forward filter to the modified received signal $\mathbf{R}^{i}[f]$,

$$
Y_{i}[f]=\mathbf{W}_{i}^{H}[f] \mathbf{R}^{i}[f],
$$

where $(\cdot)^{H}$ denotes the conjugate transpose operation. With the coefficient $\mathbf{b}_{i}^{\mathrm{FBF}}=\left[b_{i, 1}^{\mathrm{FBF}}, \ldots, b_{i, L}^{\mathrm{FBF}}\right]^{T}$ of the feedback filter, the soft output of the MISO-DFE for the $i$ th substream at the $j$ th stage is

$$
m_{i}^{j}[n]=y_{i}[n]+\sum_{l=1}^{L} b_{i, l}^{\mathrm{FBF}} \hat{s}_{i}^{j-1}[n-l], \quad n=0,1, \ldots, T-1,
$$

where $y_{i}[n]$ is the inverse DFT of $Y_{i}[f]$ and the feedback signal $\hat{s}_{i}^{j-1}[n]$ is defined as

$$
\hat{s}_{i}^{j-1}[n]= \begin{cases}\mathrm{PN}_{i, L-|n|}, & -L \leq n \leq-1 \\ \hat{d}_{i}^{j-1}[n], & 0 \leq n \leq T-1\end{cases}
$$




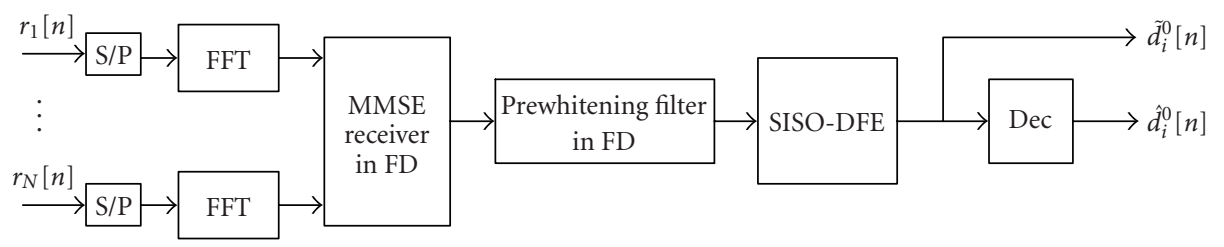

FIgURE 4: The initialization algorithm.

The mean square error of the $i$ th substream is

$$
J_{i, \mathrm{MMSE}}=E\left\{\left|m_{i}^{j}[n]-d[n]\right|^{2}\right\} .
$$

Assuming the previous tentative hard decisions are all correct, the mean square error can be expressed as follows:

$$
J_{i, \mathrm{MMSE}}=E\left\{\left|y_{i}[n]+\sum_{l=1}^{L} b_{i, l}^{\mathrm{FBF}} s_{i}[n-l]-s_{i}[n]\right|^{2}\right\} .
$$

It can be expressed in FD as

$$
\begin{gathered}
J_{i, \mathrm{MMSE}}=\frac{1}{T+L} \sum_{f=0}^{T+L-1}\left\{\sigma_{d}^{2}\left|\mathbf{W}_{i}^{H}[f](\mathbf{H}[f])_{i}+B_{i}[f]-1\right|^{2}\right. \\
\left.+\sigma_{v}^{2} \mathbf{W}_{i}^{H}[f] \mathbf{W}_{i}[f]\right\},
\end{gathered}
$$

where $B_{i}^{\mathrm{FBF}}[f]$ is the DFT of $\mathbf{b}_{i}^{\mathrm{FBF}}$ and $\sigma_{d}^{2}$ denotes the power of the transmitted symbol. Applying the gradient method to (13), we obtain

$$
\frac{\partial J_{i, \mathrm{MMSE}}}{\partial \mathbf{W}_{i}[f]}=0
$$

Therefore, the weight vector of the forward filter can be expressed as follows:

$$
\begin{aligned}
& \mathbf{W}_{i}[f] \\
& =\left[(\mathbf{H}[f])_{i}(\mathbf{H}[f])_{i}^{H}+\frac{\sigma_{v}^{2}}{\sigma_{d}^{2} \mathbf{I}}\right]^{-1}(\mathbf{H}[f])_{i}\left(1-B_{i}^{* \mathrm{FBF}}[f]\right),
\end{aligned}
$$

where ${ }^{*}$ denotes the conjugate operation and I denotes an indentity matrix. With the matrix inversion lemma in (A.2), the weight vector of the forward filter can be computed with low complexity when $\mathbf{b}_{i}^{\mathrm{FBF}}$ is known. From (13) and (15), the mean square error can be rewritten as follows:

$$
\begin{aligned}
& J_{i, \mathrm{MMSE}} \\
& =\frac{1}{T+L} \sigma_{v}^{2} \sum_{f=0}^{T+L-1} \frac{\left|1-B_{i}^{\mathrm{FBF}}[f]\right|^{2}}{\sigma_{v}^{2} / \sigma_{d}^{2}+\left\|(\mathbf{H}[f])_{i}\right\|^{2}},
\end{aligned}
$$

where $\|\cdot\|$ denotes the Frobenius norm. The derivation of (16) is given in the appendix. Applying the gradient method to (16), the optimum coefficient $\mathbf{b}_{i}^{\mathrm{FBF}}$ of the feedback filter in the MMSE sense is

$$
\mathbf{b}_{i}^{\mathrm{FBF}}=\mathbf{R}_{i}^{-1} \mathbf{a}_{i}
$$

where

$$
\begin{aligned}
{\left[\mathbf{R}_{i}\right]_{k, l} } & =\sum_{f=0}^{T+L-1} \frac{e^{-j 2 \pi f(l-k) /(T+L)}}{\left\|(\mathbf{H}[f])_{i}\right\|^{2}+\sigma_{v}^{2} / \sigma_{d}^{2}}, \\
{\left[\mathbf{a}_{i}\right]_{k} } & =\sum_{f=0}^{T+L-1} \frac{e^{j 2 \pi f k /(T+L)}}{\left\|(\mathbf{H}[f])_{i}\right\|^{2}+\sigma_{v}^{2} / \sigma_{d}^{2}} .
\end{aligned}
$$

Note that $\mathbf{R}_{i}$ is a Toeplitz matrix. Therefore, the low complexity algorithm in [15] can be utilized to compute the inverse of $\mathbf{R}_{i}$. Meanwhile, the matrix $\mathbf{R}_{i}$ and the column vector $\mathbf{a}_{i}$ can be computed using FFT algorithm. Comparing the MISO-DFE derived above with the SISO-DFE in [5], we can see that it is a generalization of a SISO-DFE. If channel frequency response $(\mathbf{H}[f])_{i}$ is a scalar, a MISO-DFE will be reduced to a SISO-DFE. With the previous tentative soft decision $\tilde{d}_{i}^{j-1}[n]$ for the $i$ th substream at the $(j-1)$ th stage, the soft decision for the $i$ th substream at the $j$ th stage is

$$
\tilde{d}_{i}^{j}[n]=p_{j} m_{i}^{j}[n]+\left(1-p_{j}\right) \tilde{d}_{i}^{j-1}[n],
$$

where $p_{j}$ is the partial decision feedback parameter at the $j$ th stage. Therefore, the tentative hard decision for the $i$ th substream at the $j$ th stage is

$$
\hat{d}_{i}^{j}[n]=\operatorname{Dec}\left(\tilde{d}_{i}^{j-1}[n]\right),
$$

where $\operatorname{Dec}(\cdot)$ denotes slicing operation appropriate to the constellation. Since tentative decisions at the early stage are less reliable than those at the later stage, the partial decision feedback parameter should increase as the detection stage continues. Here the PPIC idea stemming from the multiuser detector is introduced to reduce the effect of error propagation. Applying the above detection procedure to the $i$ th substream, MIMO frequency-selective channels are reduced to equivalent parallel single-input multiple-output frequencyselective channels. Therefore, the initialization complexity of the proposed equalizer is lower than that of the MIMO-DFE in [12] and the MIMO-OFDM in [11].

\section{INITIALIZATION}

In this section, an algorithm is introduced to obtain tentative hard and soft decisions for initialization. The initialization scheme is presented in Figure 4. From (5), the weight matrix for $M$ substreams in MMSE sense is

$$
\mathbf{W}^{0}[f]=\sigma_{d}^{2} \mathbf{H}[f]\left(\sigma_{d}^{2} \mathbf{H}[f](\mathbf{H}[f])^{H}+\sigma_{v}^{2}\right)^{-1} .
$$


TABLE 1: Complexity comparison of the equalizers design for MIMO frequency-selective channel in the case $M=N$.

\begin{tabular}{l|ll}
\hline & Matrix inversion & FFT operation \\
\hline MIMO-OFDM & $\left(2 M^{4}+2 M^{3}\right) T$ & Null \\
\hline SC-MIMO-DFE & $(T+L)^{3} M^{3} / 3+2 M^{3}(T+L)^{2}+M^{3} L$ & $M L(T+L) / 2\left(\log _{2}(T+L)-1\right)$ \\
SC-FDLE & $7(T+L) M^{3} / 3$ & Null \\
SC-FDLE-PIC & $7 M^{3} / 3(T+L)+M^{2}(T+L)$ & Null \\
SC-PMDFE & $17 M^{3} / 6(T+L)+6 M L^{2}$ & $M(T+L)\left(\log _{2}(T+L)-1\right)$ \\
\hline
\end{tabular}

TABLe 2: Complexity of signal detection for MIMO frequency-selective channel in the case $M=N$.

\begin{tabular}{|c|c|c|c|c|c|}
\hline & FFT & IFFT & PIC/PPIC & FFF & FBF \\
\hline MIMO-OFDM & $M T / 2\left(\log _{2}(T)-1\right)$ & Null & Null & $M^{2} T$ & Null \\
\hline SC-MIMO-DFE & $\begin{array}{l}M(T+L) / 2 \\
\times\left(\log _{2}(T+L)-1\right)\end{array}$ & $\begin{array}{l}M(T+L) / 2 \\
\times\left(\log _{2}(T+L)-1\right)\end{array}$ & Null & $M^{2}(T+L)$ & $L M^{2}(T+L)$ \\
\hline SC-FDLE & $\begin{array}{l}M(T+L) / 2 \\
\times\left(\log _{2}(T+L)-1\right)\end{array}$ & $\begin{array}{l}M(T+L) / 2 \\
\times\left(\log _{2}(T+L)-1\right)\end{array}$ & Null & $M^{2}(T+L)$ & Null \\
\hline SC-FDLE-PIC & $\begin{array}{l}M(T+L) \\
\times\left(\log _{2}(T+L)-1\right)\end{array}$ & $\begin{array}{l}M(T+L) \\
\times\left(\log _{2}(T+L)-1\right)\end{array}$ & $M^{2}(M-1)(T+L)$ & $2 M^{2}(T+L)$ & Null \\
\hline SC-PMDFE & $\begin{array}{l}\left(L_{s}+1\right) M(T+L) \\
\times\left(\log _{2}(T+L)-1\right)\end{array}$ & $\begin{array}{l}\left(L_{s}+1\right) M(T+L) \\
\times\left(\log _{2}(T+L)-1\right)\end{array}$ & $L_{s} M^{2}(M-1)(T+L)$ & $\begin{array}{l}\left(L_{s}+1\right) M^{2}(T+L) \\
+M(T+L)\end{array}$ & $T L M L_{s}+L M T$ \\
\hline
\end{tabular}

Then, the estimate $\mathbf{Y}^{0}[f]=\left[Y_{1}^{0}[f], \ldots, Y_{M}^{0}[f]\right]^{T}$ of each substream in FD at the $f$ th subchannel is

$$
\mathbf{Y}^{0}[f]=\left(\mathbf{W}^{0}[f]\right)^{H} \mathbf{R}[f]
$$

Therefore, the estimate for the $i$ th substream at the $f$ th subchannel is

$$
Y_{i}^{0}[f]=G_{i}^{0}[f] S_{i}[f]+V_{i}^{0}[f]
$$

where

$$
\begin{gathered}
G_{i}^{0}[f]=\left(\mathbf{W}^{0}[f]\right)_{i}^{H}(\mathbf{H}[f])_{i}, \\
V_{i}^{0}[f]=\sum_{t=1, t \neq i}^{M}\left[\left(\mathbf{W}^{0}[f]\right)_{i}\right]^{H}(\mathbf{H}[f])_{t}+\left[\left(\mathbf{W}^{0}[f]\right)_{i}\right]^{H} \mathbf{V}[f] .
\end{gathered}
$$

The colored noise $V_{i}^{0}[f]$ will degrade the performance of the DFE. Therefore, a prewhitening filter is required for each substream in FD. The output of the prewhitening filter is

$$
\begin{aligned}
& \tilde{Y}_{i}^{0}[f] \\
& =\frac{Y_{i}^{0}[f]}{\sum_{t=1, t \neq i}^{M} \sigma_{d}^{2}\left|\left[\left(\mathbf{W}^{0}[f]\right)_{i}\right]^{H}(\mathbf{H}[f])_{t}\right|^{2} / \sigma_{v}^{2}+\left\|\left(\mathbf{W}^{0}[f]\right)_{i}\right\|^{2}} \\
& =\tilde{G}_{i}^{0}[f] S_{i}[f]+\tilde{V}_{i}^{0}[f],
\end{aligned}
$$

where the equivalent channel frequency response $\tilde{G}_{i}^{0}[f]$ is defined as

$$
\begin{aligned}
& \tilde{G}_{i}^{0}[f] \\
& =\frac{G_{i}^{0}[f]}{\sum_{t=1, t \neq i}^{M} \sigma_{d}^{2}\left|\left[\left(\mathbf{W}^{0}[f]\right)_{i}\right]^{H}(\mathbf{H}[f])_{t}\right|^{2} / \sigma_{v}^{2}+\left\|\left(\mathbf{W}^{0}[f]\right)_{i}\right\|^{2}},
\end{aligned}
$$

and the interference plus noise $\tilde{V}_{i}^{0}[f]$ is

$$
\begin{aligned}
& \tilde{V}_{i}^{0}[f] \\
& =\frac{V_{i}^{0}[f]}{\sum_{t=1, t \neq i}^{M} \sigma_{d}^{2}\left|\left[\left(\mathbf{W}^{0}[f]\right)_{i}\right]^{H}(\mathbf{H}[f])_{t}\right|^{2} / \sigma_{v}^{2}+\left\|\left(\mathbf{W}^{0}[f]\right)_{t}\right\|^{2}} .
\end{aligned}
$$

With the same derivation as in [5], the optimum coefficients of the DFE for the equivalent SISO frequency-selective channel can be computed with low complexity, and initial tentative hard and soft decisions can be obtained.

\section{COMPLEXITY EVALUATION}

In this section, the complexity of existing schemes is compared in terms of complex multiplications. The data block size of MIMO-OFDM is $T$ and the length of the prefix of MIMO-OFDM is $L . B$ is the span of the feedback filter of the MIMO-DFE. $L_{s}$ denotes the number of the total detection stages of the PMDFE.

From Table 1, we can see that the initialization complexity of the MIMO-DFE [10] is too high to be implemented. 
TABLE 3: Channel profile.

\begin{tabular}{ccc}
\hline Tap index & Tap delay $(\mathrm{ns})$ & Relative power $(\mathrm{dB})$ \\
\hline 1 & 0 & -3.2 \\
2 & 100 & -5 \\
3 & 400 & -4.5 \\
4 & 500 & -3.6 \\
5 & 700 & -3.9 \\
6 & 800 & 0 \\
7 & 950 & -3 \\
8 & 1200 & -1.2 \\
9 & 1350 & -5 \\
10 & 1450 & -3.5 \\
\hline
\end{tabular}

The initialization complexity of the MIMO-OFDM is also very high and is mainly due to the expected SINR ordering.

From Table 2, it can be observed that the complexity of signal detection in various schemes mainly comes from the FFT and IFFT operations. Simulation results in the next section show that satisfactory performance can be achieved through three stages. MIMO-OFDM system has the lowest complexity of signal detection among these schemes.

\section{SIMULATION RESULTS}

The performance of various schemes is evaluated for QPSK modulation over a ten-tap channel in Table 3. Each tap is independent Rayleigh faded and quasistatic. There is no channel coding. The bit error rates (BERs) of various schemes are given versus the averaged received SNR per receiver antenna. The perfect channel information is assumed to be known at the receiver.

In Figure 5, the performance of the PMDFE with partial decision feedback parameters $p_{1}=0.7, p_{2}=0.8, p_{3}=0.9$, and $p_{4}=0.95$ is given for space-time architecture $(2 \mathrm{Tx}$, $2 \mathrm{Rx})$. If the target BER is $10^{-2}$, at the first stage about $2.0 \mathrm{~dB}$ gain can be obtained over the initial stage. Through, four stages, $2.7 \mathrm{~dB}$ gain can be obtained compared to the initial stage. From Figure 5, we can see that satisfactory performance of the PMDFE can be achieved through three stages.

In Figure 6, the performance comparison of the PMDFE with different partial decision feedback parameters is presented for $(2 \mathrm{Tx}, 2 \mathrm{Rx})$. When partial decision feedback parameters are $p_{1}=0.7, p_{2}=0.8, p_{3}=0.9$, and $p_{4}=0.95$, the PMDFE is robust against error propagation. Therefore, partial decision feedback parameters should increase as the stage continues. Research results in $[16,17]$ can be introduced to design optimum partial decision feedback parameters.

In Figure 7, the performance comparison of the PMDFE, the MIMO-OFDM [11], the FDLE, and the FDLE-PIC [12] is given for $(2 \mathrm{Tx}, 2 \mathrm{Rx})$. It can be seen that the proposed PMDFE with $p_{1}=0.7, p_{2}=0.8, p_{3}=0.9$, and $p_{4}=0.95$ has the best performance. If the target BER is $10^{-2}$, the PMDFE

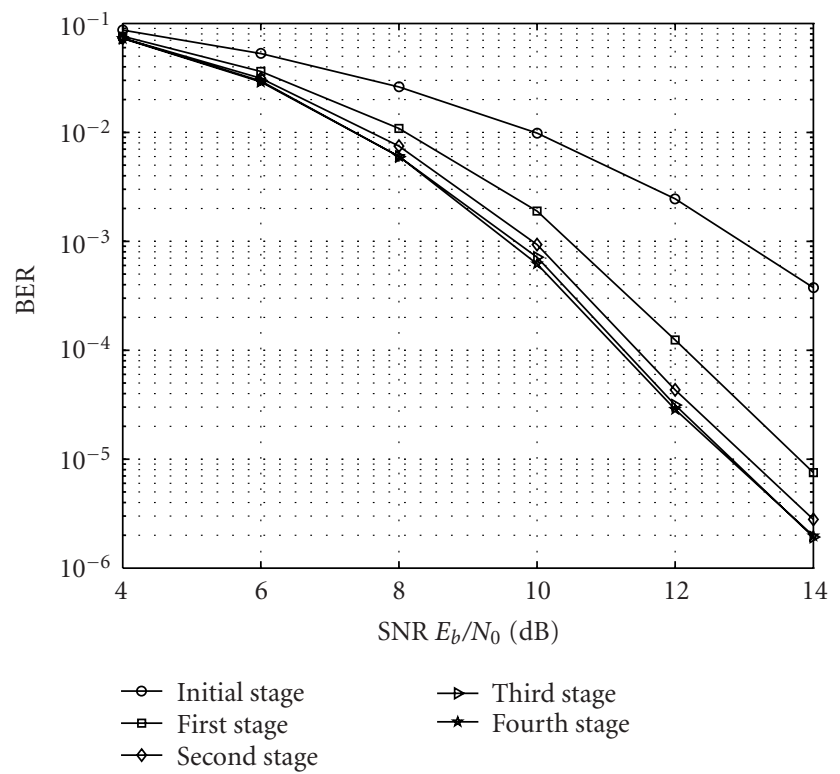

FIgure 5: The performance of the PMDFE with partial decision feedback parameters $p_{1}=0.7, p_{2}=0.8, p_{3}=0.9$, and $p_{4}=0.95$ over $(2 \mathrm{Tx}, 2 \mathrm{Rx})$.

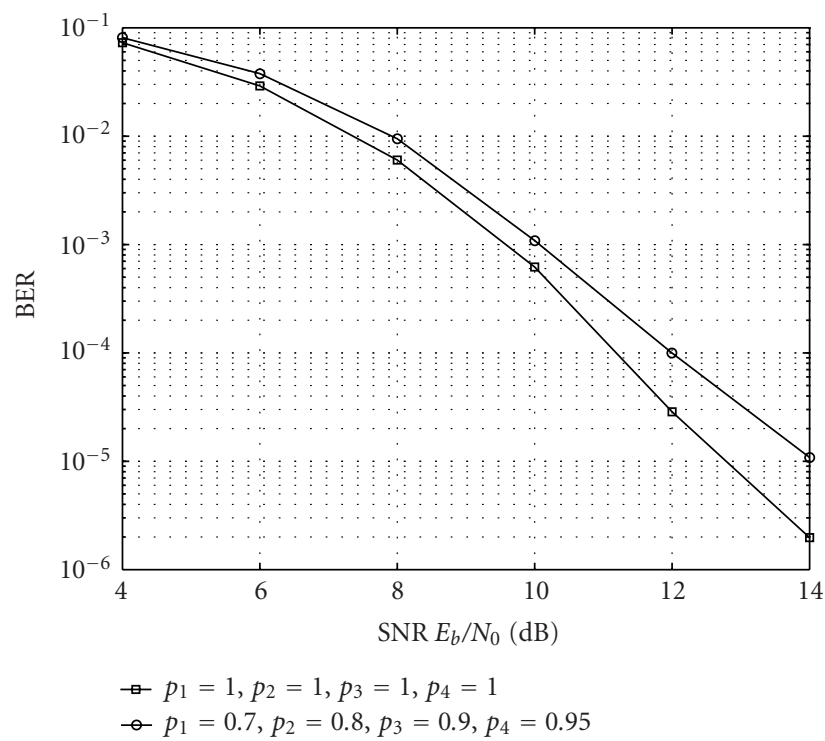

Figure 6: The performance comparison between different partial decision feedback parameters over (2Tx, 2Rx).

can obtain about $4.4 \mathrm{~dB}$ gain over the MIMO-OFDM, $2.1 \mathrm{~dB}$ gain over the FDLE-PIC, and $3.9 \mathrm{~dB}$ gain over the FDLE. As shown in [11], the MIMO-OFDM divides frequencyselective channels into many narrow parallel flat fading channels. At each subchannel, a V-BLAST processor [9] is utilized to detect the desired signal. Therefore, there does not exist multipath diversity or frequency diversity in MIMO-OFDM system. Certainly, MIMO-OFDM scheme with space-time block coding will introduce more spatial diversity to mitigate 


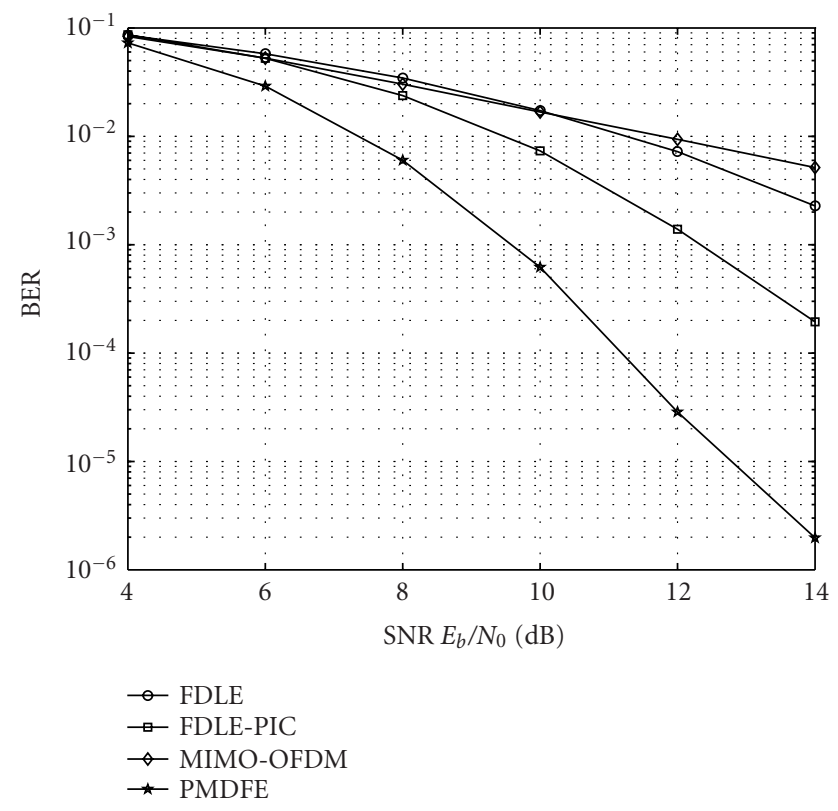

FIgURE 7: The performance comparison of the PMDFE with partial decision feedback parameters $p_{1}=0.7, p_{2}=0.8, p_{3}=0.9$, and $p_{4}=0.95$, the MIMO-OFDM, the FDLE, and the FDLE-PIC over (2Tx, 2Rx).

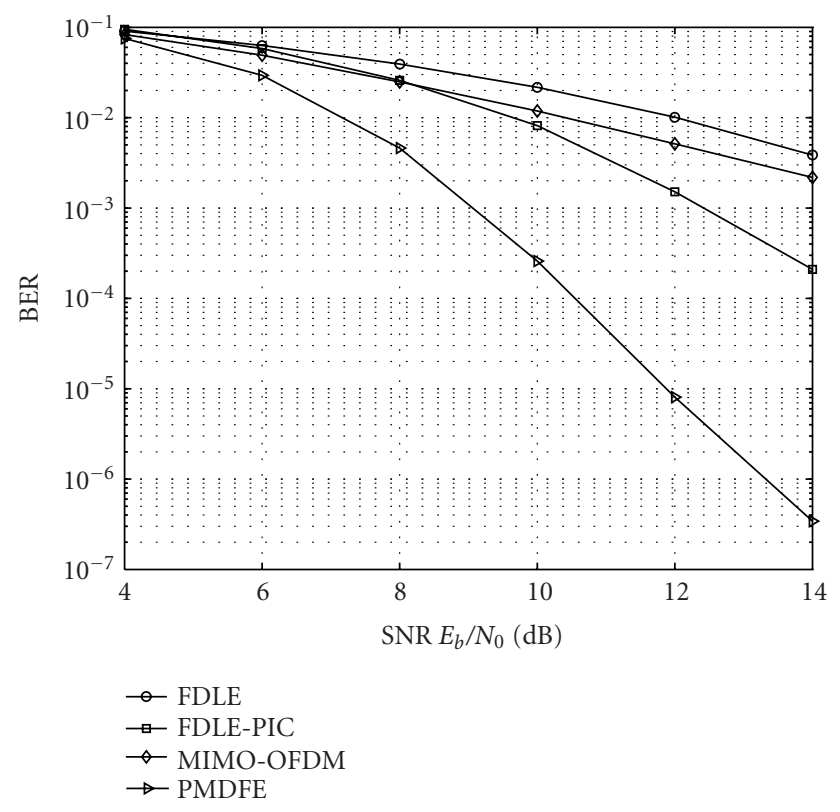

FIGURE 8: The performance comparison of the PMDFE with partial decision feedback parameters $p_{1}=0.7, p_{2}=0.8, p_{3}=0.9$, and $p_{4}=0.95$, the MIMO-OFDM, the FDLE, and the FDLE-PIC over (3Tx, 3Rx).

the fading. However, as proved in [18, 19], space-time block coding will decrease the capacity of MIMO channels. The performance of the FDLE with one-stage PIC is still limited by CCI and ISI. The proposed PMDFE can achieve multipath

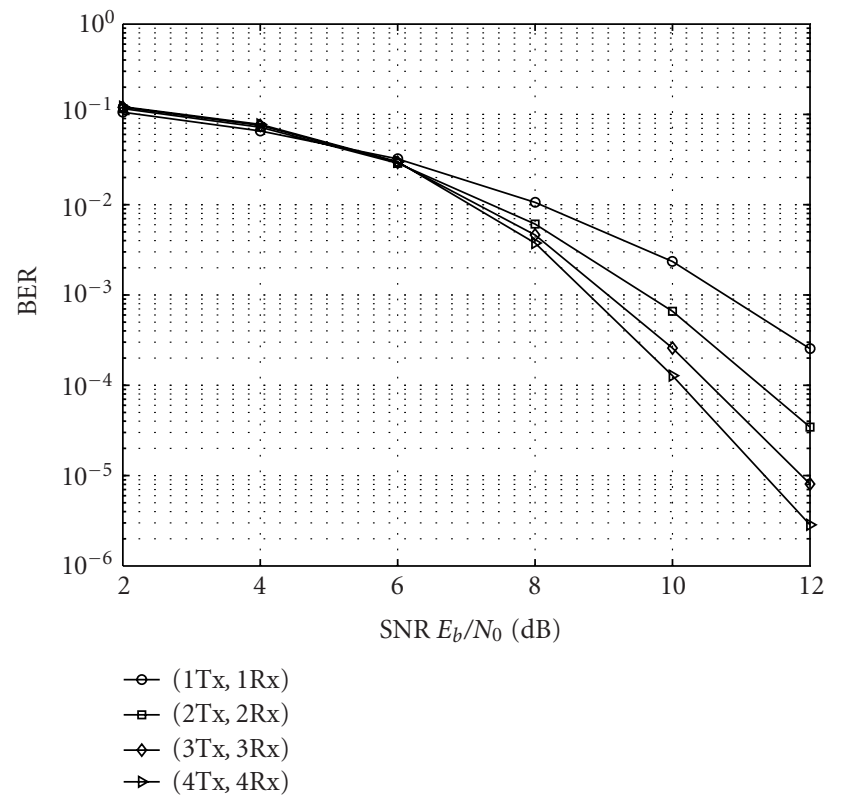

FIGURE 9: The performance of PMDFE with partial decision feedback parameters $p_{1}=0.7, p_{2}=0.8, p_{3}=0.9$, and $p_{4}=0.95$ over (1Tx, 1Rx), (2Tx, 2Rx), (3Tx, 3Rx), and (4Tx, 4Rx).

and spatial diversity simultaneously. Moreover, the great improvement is obtained and the error propagation is reduced when the detection stage continues.

In Figure 8, the performance comparison of the PMDFE, the MIMO-OFDM [11], the FDLE, and the FDLE-PIC in [12] is given for $(3 \mathrm{Tx}, 3 \mathrm{Rx})$. If the target $\mathrm{BER}$ is $10^{-2}$, the PMDFE can obtain about $3.2 \mathrm{~dB}$ gain over the MIMOOFDM, $2.5 \mathrm{~dB}$ gain over the FDLE-PIC, and $4.8 \mathrm{~dB}$ gain over the FDLE.

In Figure 9, the BER performance of the PMDFE with partial decision feedback parameters $p_{1}=0.7, p_{2}=0.8$, $p_{3}=0.9$, and $p_{4}=0.95$ over $(1 \mathrm{Tx}, 1 \mathrm{Rx}),(2 \mathrm{Tx}, 2 \mathrm{Rx}),(3 \mathrm{Tx}$, $3 \mathrm{Rx})$, and $(4 \mathrm{Tx}, 4 \mathrm{Rx})$ are compared. When the space-time architecture is reduced to $(1 \mathrm{Tx}, 1 \mathrm{Rx})$, the MISO-DFE is reduced to a SISO-DFE [5]. The performance of the PMDFE will be improved at a high SNR as the number of the transmitter or receiver antennas increases.

\section{CONCLUSION}

In this paper, a PMDFE is proposed for single-carrier layered space-time systems with the fixed CP. It is equivalent to a DFE in SD combined with a MISO-DFE in FD and TD. It can achieve better performance than the MIMO-OFDM, the FDLE, and the FDLE-PIC.

\section{APPENDIX}

For notation simplicity, we define

$$
\mathbf{W}=\mathbf{W}_{i}[f], \quad B=B_{i}[f], \quad \mathbf{H}=(\mathbf{H}[f])_{i} .
$$


With the matrix inversion lemma,

$$
\left(\mathbf{A}+\mathbf{a} \mathbf{b}^{H}\right)^{-1}=\mathbf{A}^{-1}-\frac{\mathbf{A}^{-1} \mathbf{a b}^{H} \mathbf{A}^{-1}}{c}, \quad c=1+\mathbf{b}^{H} \mathbf{A}^{-1} \mathbf{a} .
$$

Therefore, we have

$$
\left(\frac{\sigma_{v}^{2}}{\sigma_{d}^{2} \mathbf{I}}+\mathbf{H} \mathbf{H}^{H}\right)^{-1}=\frac{\sigma_{d}^{2}}{\sigma_{v}^{2}}\left[\mathbf{I}-\frac{\sigma_{d}^{2} \mathbf{H} \mathbf{H}^{H}}{\sigma_{v}^{2} c}\right],
$$

where

$$
c=1+\frac{\sigma_{d}^{2}\|\mathbf{H}\|^{2}}{\sigma_{v}^{2}} .
$$

We compute the first term of the summation in (13). Substituting (15) into (13), we have that

$$
\begin{aligned}
& \sigma_{d}^{2}\left|\mathbf{W}^{H} \mathbf{H}+B-1\right|^{2} \\
&=\sigma_{d}^{2}\left|\mathbf{H}^{H}\left(\mathbf{H} \mathbf{H}^{H}+\frac{\sigma_{v}^{2}}{\sigma_{d}^{2} \mathbf{I}}\right)^{-1} \mathbf{H}(1-B)+(B-1)\right|^{2} .
\end{aligned}
$$

From (A.3), we have

$$
\begin{aligned}
& \sigma_{v}^{2} \mathbf{W}^{H} \mathbf{W} \\
& =\sigma_{d}^{2}\left|\frac{\sigma_{d}^{2}}{\sigma_{v}^{2}}\left[\|\mathbf{H}\|^{2}-\frac{\|\mathbf{H}\|^{4} \sigma_{d}^{2}}{c \sigma_{v}^{2}}\right](1-B)+(B-1)\right|^{2} \\
& =\frac{\sigma_{d}^{2}}{c^{2}}|1-B|^{2} .
\end{aligned}
$$

With the same derivation, the second term of the summation in (13) can expressed as

$$
\begin{aligned}
\sigma_{v}^{2} \mathbf{W}^{H} \mathbf{W} \\
=\frac{\sigma_{d}^{4}}{\sigma_{v}^{2}} \mathbf{H}^{H}\left(\mathbf{I}-\frac{\sigma_{d}^{2} \mathbf{H} \mathbf{H}^{H}}{c \sigma_{v}^{2}}\right)\left(\mathbf{I}-\frac{\sigma_{d}^{2} \mathbf{H} \mathbf{H}^{H}}{c \sigma_{v}^{2}}\right) \mathbf{H}|1-B|^{2} \\
=\frac{\sigma_{d}^{4}}{\sigma_{v}^{2} c^{2}}|1-B|^{2}\|\mathbf{H}\|^{2} .
\end{aligned}
$$

With (A.6) and (A.7), we have that

$$
\begin{aligned}
\sigma_{d}^{2}\left|\mathbf{W}^{H} \mathbf{H}+B-1\right|^{2}+\sigma_{v}^{2} \mathbf{W}^{H} \mathbf{W} \\
=\frac{\sigma_{d}^{2}|1-B|^{2}}{c}=\frac{\sigma_{v}^{2}|1-B|^{2}}{\sigma_{v}^{2} / \sigma_{d}^{2}+\|\mathbf{H}\|^{2}} .
\end{aligned}
$$

\section{ACKNOWLEDGMENTS}

This work was supported by Nokia Mobile Phones and Chinese 863 high technology development project (2002AA123031). This paper was presented in part at the 14th IEEE International Symposium on Personal, Indoor and Mobile Radio Communications (PIMRC), September 2003.

\section{REFERENCES}

[1] M. Rohling, T. May, K. Bruninghaus, and R. Grunheid, "Broad-band OFDM radio transmission for multimedia applications," Proceedings of the IEEE, vol. 87, no. 10, pp. 17781789, 1999.

[2] M. Huemer, L. Reindl, A. Springer, and R. Weigel, "Frequency domain equalization of linear polyphase channels," in Proc. 51st IEEE Vehicular Technology Conference (VTC '00), vol. 3, pp. 1698-1702, Tokyo, Japan, May 2000.

[3] H. Sari, G. Karam, and I. Jeanclaude, "Transmission techniques for digital terrestrial TV broadcasting," IEEE Communications Magazine, vol. 33, no. 2, pp. 100-109, 1995.

[4] A. Czylwik, "Comparison between adaptive OFDM and single carrier modulation with frequency domain equalization," in Proc. 47th IEEE Vehicular Technology Conference (VTC '97), vol. 2, pp. 865-869, Phoenix, Ariz, USA, May 1997.

[5] D. Falconer, S. L. Ariyavisitakul, A. Benyamin-Seeyar, and B. Eidson, "Frequency domain equalization for single-carrier broadband wireless systems," IEEE Communications Magazine, vol. 40, no. 4, pp. 58-66, 2002.

[6] N. Benvenuto and S. Tomasin, "On the comparison between OFDM and single carrier modulation with a DFE using a frequency-domain feedforward filter," IEEE Trans. Communications, vol. 50, no. 6, pp. 947-955, 2002.

[7] H. Witschnig, T. Mayer, A. Springer, et al., "A different look on cyclic prefix for SC/FDE," in Proc. 13th IEEE International Symposium on Personal, Indoor and Mobile Radio Communications (PIMRC '02), vol. 2, pp. 824-828, Lisbon, Portugal, September 2002.

[8] G. J. Foschini, "Layered space-time architecture for wireless communication in a fading environment when using multiple antennas," Bell Labs Technical Journal, vol. 1, no. 2, pp. 41-59, 1996.

[9] G. D. Golden, C. J. Foschini, R. A. Valenzuela, and P. W. Wolniansky, "Detection algorithm and initial laboratory results using V-BLAST space-time communication architecture," Electronics Letters, vol. 35, no. 1, pp. 14-16, 1999.

[10] A. Lozano and C. Papadias, "Layered space-time receivers for frequency-selective wireless channels," IEEE Trans. Communications, vol. 50, no. 1, pp. 65-73, 2002.

[11] L. Giangaspero, L. Agarossi, G. Paltenghi, S. Okamura, M. Okada, and S. Komaki, "Co-channel interference cancellation based on MIMO OFDM systems," IEEE Wireless Communications, vol. 9, no. 6, pp. 8-17, 2002.

[12] X. Zhu and R. D. Murch, "Novel frequency-domain equalization architectures for a single-carrier wireless MIMO system," in Proc. 56th IEEE Vehicular Technology Conference (VTC'02), vol. 2, pp. 874-878, Vancouver, BC, Canada, September 2002.

[13] D. Divsalar, M. K. Simon, and D. Raphaeli, "Improved parallel interference cancellation for CDMA," IEEE Trans. Communications, vol. 46, no. 2, pp. 258-268, 1998.

[14] T. Walzman and M. Schwartz, "Automatic equalization using the discrete frequency domain," IEEE Transactions on Information Theory, vol. 19, no. 1, pp. 59-68, 1973.

[15] G. H. Golub and C. F. Van Loan, Matrix Computations, John Hopkins University Press, Baltimore, Md, USA, 3rd edition, 1996.

[16] G. Xue, J. Weng, T. Le-Ngoc, and S. Tahar, "Adaptive multistage parallel interference cancellation for CDMA," IEEE Journal on Selected Areas in Communications, vol. 17, no. 10, pp. 1815-1827, 1999.

[17] J.-H. Wen and Y.-F. Huang, "Fuzzy-based adaptive partial parallel interference canceller for CDMA communication systems over fading channels," IEE Proceedings Communications, vol. 149, no. 2, pp. 111-114, 2002. 
[18] B. Hassibi and B. M. Hochwald, "High-rate codes that are linear in space and time," IEEE Transactions on Information Theory, vol. 48, no. 7, pp. 1804-1824, 2002.

[19] R. W. Heath Jr. and A. J. Paulraj, "Linear dispersion codes for MIMO systems based on frame theory," IEEE Trans. Signal Processing, vol. 50, no. 10, pp. 2429-2441, 2002.

Jing Xu was born in Wuxi, China, in 1975. He received his M.S. degrees in electronic engineering from Jilin University, Changchun, China, in 2001. Now he is a Ph.D. student at the National Mobile Communications Research Laboratory, Southeast University in Nanjing. His main research interests include equalization, CDMA system, and signal processing.

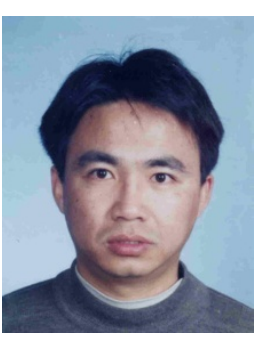

Haifeng Wang was born in Shanghai, China, in 1969. He received his M.S. and Ph.D. degrees, both in electronic engineering from Tampere University of Technology, Tampere, Finland, in 1993 and 2000, respectively. Since 1995, he has been working in Nokia Mobile Phones, Oulu, Finland. Currently, he is a Research Specialist and a Project Manager for standardization and research on $3 \mathrm{G}$ evolution. His main research

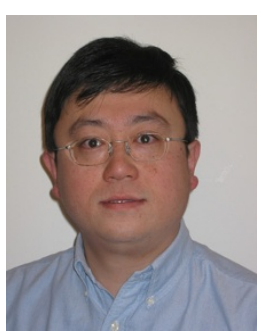
interests are in the fields of adaptive and robust signal processing, antenna array processing, and mobile communication systems. He has published over 60 research papers and holds 7 pending patents.

Shixin Cheng graduated from Nanjing Institute of Technology in 1958 and finished his graduate study at the former Leningrad Telecommunications Institute of St. Petersburg. He was an Honorary Research Fellow of Birmingham University of UK from 1979 to 1982 and a Visiting Professor at Aichi University, Japan, Concordia University, Canada, and ETH, Switzerland, in the period from 1985 to 2002 . He is now a Pro-

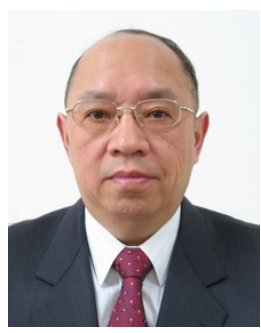
fessor at Southeast University, China, and Vice Chairman of Academic Committee for the National Mobile Communications Research Laboratory. His research interests include wireless communication theory and the future mobile communications technology.

Ming Chen received his B.S., M.S., and Ph.D. degrees from the Mathematics Department, Nanjing University, Nanjing, China, in 1990, 1993, and 1996, respectively. In July 1996, he came to National Mobile Communications Research Laboratory, Southeast University, Nanjing, to be a Lecturer. From April 1998 to March 2003, he was an Associate Professor and from April 2003 till now, he has been a Professor at the

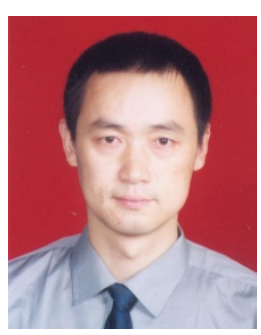
laboratory. His research interests include digital communication theory and signal processing for wireless communication systems with emphasis on multiuser detection, adaptive antenna, spacetime coding, and radio resource management. 